the different inclusion and exclusion criteria, we obtained 1156 patients, 733 CC patients and 423 non-CC patients. Subsequently, and after analyzing the first results, we decided to homogenize our database by means of a PMS analysis, by this way, we obtained a new balanced population of 374 patients (187 CC patients and 187 non-CC patients).

Results In the general population, patients with CC present a $72 \%$ reduction in the risk of relapse compared to non-CC patients (HR: 0.28 95\% CI (0.17-0.46) p = 0.000) and a 90\% reduction in the risk of death (HR: 0.10 95\% CI $(0.03-$ 0.33) $\mathrm{p}=0.000)$, these differences may be due to the fact that both populations present differences.

After homogenizing our population using the PMS, we obtained that the reduction in the risk of relapse was $65 \%$ for patients who have CC (HR: 0.35 CI 95\% (0.16-0.75) p $=0.007)$ and $75 \%$ for the risk of death for the same cohort (HR: 0.25 95\% CI (0.07-0.90) p = 0.033).

Regarding the secondary objectives, we observed that the CC seems to have a protective effect in tumors between 2-4 cm (HR: 0.33 95\% CI $(0.11-0.99) \mathrm{p}=0.049)$. This same protective effect is observed in patients operated on by laparoscopy (HR: $0.3595 \%$ CI $(0.14-0.89) \mathrm{p}=0.028)$. Finally, the MIS patients who have CC do not present differences compared to those operated by the open approach, whether they are conized or non-conized (Log-Rank p $=0.439$ and LogRank p $=0.346)$.

Conclusion Patients undergoing CC have a significantly lower risk of relapse and death, this effect is more evident in those patients with $2-4 \mathrm{~cm}$ tumors or in those who are operated under MIS.

Disclosures I have nothing to disclose.

\section{DESIGN AND VALIDATION OF A RECURRENCE RISK PREDICTING SCORE IN EARLY STAGE CERVICAL CANCER AFTER RADICAL HYSTERECTOMY}

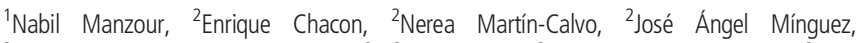
${ }^{2}$ Teresa Castellano, Daniel Vazquez ${ }^{2}$, ${ }^{2}$ Diego Salas, ${ }^{2}$ Antonio Gonzalez-Martin, ${ }^{3}$ Juan Luis Alcazar, '2Luis M Chiva. 'Clínica Universidad de Navarra; Clinica Universidad de Navarra; Gynecology; ${ }^{2}$ Clinica Universidad de Navarra; ${ }^{3}$ Clínica Universidad de Navarra; Clinica Universidad de Navarra
\end{abstract}

\subsection{6/ijgc-2020-ESGO.17}

Introduction/Background After the LACC trial, the scientific evidence has focused on confirming and finding the cause of why the open route presents better results than minimally invasive surgery (MIS). Even though the independent factors involved in relapse has not been studied.

\section{Methodology}

Primary objective to know the independent clinical, surgical and anatomopathological factors involved in the relapse of patients with stage IB1 cervical cancer who underwent radical hysterectomy (2013-2014).

Secondary objective To create a risk predictive index (RPI) that allows us to better select and stratify patients with a higher probability of relapse.

Methods Starting from 1272 patients from the European database belonging to the SUCCOR study and after applying the different inclusion and exclusion criteria we obtained 1156 patients. We randomly divided our sample into a test group and a validation group in a proportion of $60 \%$ to $40 \%$.
The test group was used to identify the variables independently associated with relapse and to define the relapse RPI. The RPI was applied to calculate a relapse risk score for each participant in the validation group. According to their risk of relapse, participants were classified into 3 risk groups.

Results Women who relapse are more likely to have tumours larger than $2 \mathrm{~cm}$ on imaging assessment (OR 2.15, 95\% CI 1.33- 3.5) and to undergo MIS (OR 1.61, 95\% CI 1.002.57). On the other hand, conisation is inversely associated with the risk of relapse (OR 0.31, 95\% CI 0.17- 0.60).

The AUC in the validation group for RPI is $(0.72 ; 95 \% \mathrm{CI}$ 0.65- 0.79).

Depending on their score, patients were classified at low, medium or high risk of relapse. The relapse rate observed in each group was $3.4 \%, 9.8 \%$ and $21.3 \%$ respectively.

With a median follow-up of 58 months, the mean DFS in the validation group for low, medium and high risk categories were 75.4 (95\% CI 73.8- 76.9), 75.5 (95\% CI 72.4- 78.5) and 64.1 (95\% CI 59.4- 68.9) months respectively $(\mathrm{P}<$ $0.001)$.

Conclusion Our risk predictor index proved to be valid and therefore may help to identify those patients who would benefit from adjuvant therapy and close follow-up after radical hysterectomy.

\section{SURVIVAL OUTCOMES OF PATIENTS WITH CLEAR CELL CARCINOMA CERVIX: A SINGLE INSTITUTIONAL RETROSPECTIVE ANALYSIS}

${ }^{1}$ Akhilesh CS Sudhakaran, ${ }^{2}$ Aswin Kumar, ${ }^{3}$ Francis James, ${ }^{2}$ Susan Mathews, ${ }^{2}$ John Joseph. ${ }^{1}$ Regional Cancer Centre, Trivandrum, Kerala, India; Radiation Oncology; ${ }^{2}$ Regional Cancer Centre, Trivandrum, Kerala, India; Division OF Gynec Oncology; ${ }^{3}$ Regional Cancer Centre; Regional Cencer Centre, Trivandrum, Kerala, India; Division OF Gynec Oncology

\subsection{6/ijgc-2020-ESG0.18}

Introduction/Background Cervical cancer is the most prevalent cancer and the fifth most common cause of cancer death in Indian women. Clear cell carcinoma of the cervix (CCCC) is rare, accounting for only $4 \%$ of all adenocarcinomas. CCCC can occur sporadically or in association with in-utero diethylstilbestrol (DES) exposure. There are no clear-cut treatment recommendations for the management of CCCC. Early-stage disease is usually treated by surgery and more locally advanced stages by chemoradiation followed by brachytherapy. Methodology

Aim: This study aimed to assess the survival outcomes and patterns of failure of patients with CCCC.

Settings and Design: Retrospective study done at Regional Cancer Centre, Thiruvananthapuram, Kerala, India

Material and Methods: Case records of all the patients with CCCC who were diagnosed and treated between 1995 and 2015 were reviewed for clinical, pathological and treatment characteristics.

Statistical analysis: Disease-free survival (DFS) and overall survival (OS) were estimated using the Kaplan-Meier method.

Results The diagnosis of clear cell carcinoma of the cervix was confirmed in 15 patients. The median age was 53 years. $20 \%$ of the patients were in the International Federation of Gynaecology and Obstetrics (FIGO) stage I, 60\% in stage II, $7 \%$ in Stage III and $13 \%$ in stage IVA. Stage IB and IVA patients were managed surgically, and adjuvant therapy decided based on the tumour pathology. Stage IIB and IIIB 
patients were treated with concurrent chemoradiation followed by brachytherapy. The median follow-up period was 47 months. The three-year DFS and OS were $13.3 \%$ and $13.3 \%$ respectively.

Conclusion CCCC has a poor prognosis, stage for stage compared to other histologies. The FIGO stage, tumour size, lymphovascular space invasion and pelvic node status were factors that predicted the prognosis. Adjuvant radiotherapy or chemoradiotherapy have a limited role in the treatment of this rare cancer.

Disclosures The authors have no potential conflict of interest to disclose.

\section{EFFICACY OF A MULTI-INGREDIENT CORIOLUS VERSICOLOR-BASED VAGINAL GEL IN HPV+ WOMEN OLDER THAN 40 YEARS: SUB-ANALYSIS OF PALOMA CLINICAL TRIAL}

${ }^{1}$ Yann Gaslain, ${ }^{2}$ Luis Serrano, ${ }^{3}$ Andrés Carlos López, ${ }^{2}$ Silvia González, ${ }^{4}$ Santiago Palacios, ${ }^{5}$ Damian Dexeus, ${ }^{6}$ Pluvio Coronado, ${ }^{7}$ Jesús de la Fuente, ${ }^{8}$ José Antonio López, ${ }^{9}$ Cristina Vanrell. ${ }^{1}$ Procare Health; ${ }^{2}$ Centro Médico Gabinete Velázquez; ${ }^{3}$ Hospital Quironsalud; ${ }^{4}$ Instituto Palacios Salud Y Medicina de la Mujer; ${ }^{5}$ Women's Health Institute; ${ }^{6}$ Hospital Clínico San Carlos; ${ }^{7}$ Hospital Universitario Infanta Leonor; ${ }^{8}$ Hospital General Universitario de Alicante; ${ }^{9}$ Hospital de la Santa Creu I Sant Pau

\subsection{6/ijgc-2020-ESGO.19}

Introduction/Background HPV clearance and resolution of cervical HPV-dependent lesions become difficult in peri and postmenopausal women. The objective of this sub-analysis was to evaluate the effect of the Papilocare ${ }^{\circledR}$, a multi-ingredient Coriolus versicolor-based vaginal gel, on the normalization of cervical HPV-dependent atypia (ASCUS and LSIL) and associated colposcopic alterations in women older than 40 years.

Methodology Paloma clinical trial (ClinicalTrials. gov NCT04002154) was a multicenter, randomized, open-label, parallel-group, usual practice-controlled clinical trial. Unvaccinated HPV positive women aged between 30-65 with cytology of ASCUS or LSIL and concordant colposcopic image were randomized into 3 groups: A) Papilocare ${ }^{\circledR} 1$ cannula/ day for 1 month +1 cannula/alternate days for 5 months; B) Papilocare ${ }^{\circledR} 1$ cannula/day for 3 months +1 cannula/ alternate days for 3 months; C) Control group: no treatment (usual clinical practice). Primary endpoint:\% of patients with normal cytology and concordant colposcopy after 6 months of treatment in the total population, high-risk HPV $(16,18,31,33,35,39,45,51,52,56,58,59,68)$ and very high-risk HPV (patients infected by any combination of 16, 18 and 31) subpopulations. Pap smear evaluations were blind and centrally conducted by an independent researcher at the IECM laboratory (Lugo, Spain). Papilocare ${ }^{\circledR}$ arms (A+B) were combined as treatment group.

Results A total of 41 out of 84 evaluated patients included in Paloma trial were older than 40y [mean (SD) age: 47.71 (5.56)], of which 30 and 13 were high-risk HPV and 16-18$31 \mathrm{HPV}$ patients, respectively. At 6 months, normal cytology and concordant colposcopic image was observed in 92\%, 90\% and $75 \%$ of patients treated with Papilocare ${ }^{\circledR}$ vs $50 \%, 33 \%$ and $40 \%$ of patients in control group, in the total population, and high-risk and 16-18-31 subpopulations $(\mathrm{p}=0.0066$; $\mathrm{p}=0.0031 ; \mathrm{p}=0.2929$, Fisher test) respectively.
Conclusion Papilocare ${ }^{\circledR}$ showed a robust efficacy in normalizing cervical HPV lesions in women older than 40 years old, with a statistically significant difference vs control group in the total and high-risk populations.

Disclosures

Funding Procare Health Disclosure: J.Cortés, S.Palacios, D. Dexeus, L. Serrano: Advisory/Consulting Role and Speakers at Procare Health. Y. Gaslain: CEO of Procare Health.

All other authors have declared no conflicts of interest.

\section{REAL-LIFE EFFICACY OF A MULTI-INGREDIENT CORIOLUS VERSICOLOR-BASED VAGINAL GEL IN HIGH- RISK HPV PATIENTS: INTERIM ANALYSIS}

${ }^{1}$ Yann Gaslain, ${ }^{2}$ Javier Cortés, ${ }^{3}$ Javier de Santiago, ${ }^{4}$ María Pilar Sanjuán, ${ }^{5}$ Marta Agenjo, ${ }^{2}$ Gabriel Fiol, ${ }^{6}$ Carmen Garcia, ${ }^{7}$ Marta Gurrea, ${ }^{2}$ Perla Hernández. ${ }^{1}$ Procare Health; ${ }^{2}$ Private Practice; ${ }^{3}$ Centro Oncológico MD Anderson; ${ }^{4}$ Clínica Ginemed; ${ }^{5}$ Hospital Sanitas La Zarzuela; ${ }^{6} \mathrm{Hm}$ San Francisco; ${ }^{7} \mathrm{Hospital}$ La Fe

\subsection{6/ijgc-2020-ESG0.20}

Introduction/Background Real-life studies inform on the 'effectiveness' of a treatment what is intended to do in routine circumstances. The aim of this study is to evaluate the efficacy of Papilocare ${ }^{\circledR}$ - a multi-ingredient Coriolus versicolor-based vaginal gel- on repairing high-risk (HR) HPV-dependent lowdegree cervical lesions and HR-HPV clearance in real-life practice.

Methodology Observational, multicenter, prospective, onecohort study (PAPILOBS study ClinicalTrial.gov: NCT04199260). Currently recruiting 300 vaccinated or not HPV-positive women aged $>25 \mathrm{y}$ with Pap smear of ASCUS or LSIL and concordant colposcopy during routine clinical visits in Spain. Patients are treated with Papilocare ${ }^{\circledR} 1$ cannula/ day for 21 days the first month +1 cannula/alternate days for 5 months. After this 6-month period, patients with altered cytology and/or HPV persistency are treated for a 6-month extension treatment period with the same dosage.

Interim analysis of HR-HPV patients with normal Pap smear and concordant colposcopy image (primary endpoint) and patient with HR-HPV cleared (patients with total clearance or partial clearance together with negative Pap smear and normal colposcopy) at $6 / 12$ months is presented. The study was approved by the ethical committee of Public University Hospital of Puerta de Hierro (Madrid). Informed consent was signed by all patients.

Results At 6 months, data of 148 and 146 patients for Pap smear/colposcopy and HR-HPV presence, respectively, were available. $67.6 \%$ of patients $(100 / 148)$ had negative Pap smear and concordant colposcopy. HR-HPV clearance was observed in $58.9 \%$ of patients $(86 / 146)$. Data of 46 and 44 patients included in the 6-month extension treatment period for Pap smear/colposcopy and HR-HPV presence, respectively, were available. At 12 months, 78.3\% (36/46) of patients had negative Pap smear and concordant colposcopy and HR-HPV clearance was observed in $70.5 \%$ (31/44). Considering all study period, $77 \%(114 / 148)$ and $72.6 \%(106 / 146)$ of patients repaired HR-HPV-dependent cervical lesions and cleared HRHPV, respectively.

Conclusion In this interim analysis, repairing of HR-HPVdependent low-degree cervical lesions and clearing HR-HPV, in real life conditions, was achieved after 6-month treatment 